


Original

\title{
Diferenças na aptidão física de idosos participantes de jogos adaptados e sedentários: um estudo transversal
}

\section{Differences in the Physical Fitness of Elderly Participants of Adapted Games and Sedentary: A Cross-Sectional Study}

Rodrigues et al. 
Artigo Original

Original Article

\title{
Diferenças na aptidão física de idosos participantes de jogos adaptados e sedentários: um estudo transversal
}

\section{Differences in the Physical Fitness of Elderly Participants of Adapted Games and Sedentary: A Cross-Sectional Study}

\author{
Fernanda Regina Rodrigues ${ }^{1}$ MS; Clarissa Biehl Printes ${ }^{\S 1}$ PhD; Rudnei Andrade ${ }^{2}$ MS; Ângelo José \\ Gonçalves Bós ${ }^{1} \mathrm{PhD}$
}

Recebido em: 30 de maio de 2017. Aceito em: 15 de agosto de 2017

Publicado online em: 29 de setembro de 2017.

\section{Resumo}

Introdução: A prática esportiva em idosos levar em consideração as modificações físicas mais importantes decorrentes do processo de envelhecimento.

Objetivo: Avaliar a aptidão física dos idosos participantes de uma equipe de jogos adaptados para a terceira idade (fisicamente ativos) e idosos sedentários.

Métodos: Estudo do tipo transversal para o qual 115 participantes idosos ( $>60$ anos de idade) das atividades da Universidade Aberta para Terceira Idade (UNATI) foram convidados para participar. Depois de aplicados os critérios de inclusão e de exclusão, foram divididos em Equipe Esportiva (EE) e Grupo Sedentário (GS). Para se avaliar a aptidão física foram utilizados: testes de força e resistência; velocidade, equilíbrio dinâmico; capacidade aeróbica; e flexibilidade do Senior Fitness Test (SFT). Realizou-se análise descritiva das variáveis antropométricas da altura e peso e analítica. As médias dos desempenhos nos testes foram comparadas pelo $t$ Student com teste de Bartlett quanto à normalidade da distribuição dos dados.

Resultados: Fizeram parte das análises 53 idosos: 28 no grupo EE e 25 no GS. Em todas as avaliações físicas o desempenho dentro da faixa normal e acima do normal foi mais elevado nos idosos fisicamente ativos do que nos sedentários. Entretanto, houve

Pontos-Chave Destaque

- Idosos fisicamente ativos

apresentaram-se mais

frequentemente acima do normal do que os sedentários.

- Idosos fisicamente ativos apresentaram maiores capacidade aeróbica, velocidade, agilidade e equilíbrio dinâmico do que os sedentários.

- Idosos fisicamente ativos apresentaram maior

flexibilidade de membros. diferença estatisticamente significativa somente em capacidade aeróbica, em flexibilidade de membro inferior esquerdo, flexibilidade de membros superiores e em velocidade, agilidade e equilíbrio dinâmico $(\mathrm{p}<0,05)$.

Conclusão: Idosos fisicamente ativos e sedentários apresentaram aptidão física dentro das faixas de referência dos testes, mas os fisicamente ativos (que praticavam jogos adaptados) apresentaram melhores valores, indicando que o programa de atividades físicas para idosos tem contribuído para melhorar a aptidão física dos participantes regulares, com destaque para a capacidade aeróbica que se associa à prevenção de doenças cardiovasculares e de outras doenças crônicas.

Palavras-chave: esporte adaptado, idosos, aptidão física, desempenho físico.

\footnotetext{
${ }^{\S}$ Autor correspondente: Clarissa Biehl Printes - e-mail: clarissaprintes@hotmail.com.

Afiliações: ${ }^{1}$ Instituto de Geriatria e Gerontologia da PUCRS, Porto Alegre, RS; ${ }^{2}$ Centro Universitário La Salle, Canoas, RS.
} 


\begin{abstract}
Introduction: Sports practice in elderly must to consider and focuses the most important physical changes resulting from the aging process.

Objective: To evaluate the physical fitness of the elderly participants of a team of games adapted for the elderly (physically active) and sedentary elderly.

Methods: This was a cross-sectional study in which all 115 elderly participants ( $>60$ years of age) from the activities of the University for Third Age were invited to participate. After application of the criteria for inclusion and exclusion, participants were divided into Team Sports (TS) and Sedentary Group (SG). To evaluate the physical fitness were used: Strength and endurance tests; speed, dynamic balance; aerobic capacity; and flexibility of the Senior Fitness Test (SFT). A descriptive analysis of the anthropometric variables of height and weight and analytical was performed. The means of the performances in the tests were compared by the unpaired $t$ Student with Bartlett's test regarding the normality of data distribution.
\end{abstract}

Results: Participated in this study 53 elderly, in the ST 28 and SG 25. In all physical evaluations performance within the normal and above normal range was higher in the physically active elderly than in the sedentary: 2Minute Step Test (TS 95,1 $\pm 26,62$, SG 60,8 $\pm 21,38$ ) and the 8-Foot Up-and-Go (TS 0,05 $\pm 0,008$, SG 0,13 $\pm 0,218$ ).

Conclusion: Physically active and sedentary individuals presented physical fitness within the reference ranges of the tests, but the physically active (who practiced adapted games) presented better values, indicating that the physical activity program for the elderly has contributed to improve the physical fitness of the participants and highlighted the aerobic capacity associated with the prevention of cardiovascular diseases and other chronic diseases.

Keywords: adapted sport, elderly, physical fitness, physical performance.

\title{
Diferenças na aptidão física de idosos participantes de jogos adaptados e sedentários: um estudo transversal
}

\section{Introdução}

No Brasil, a classificação quanto à idade cronológica adotada pelos órgãos governamentais é a constante no Estatuto do Idoso, que considera idoso o indivíduo com idade acima dos 60 anos(1). Segundo projeções do Instituto Brasileiro de Geografia e Estatística (IBGE), a população brasileira possui cerca de 26 milhões de pessoas com idade igual ou superior a 60 anos, o que representa aproximadamente $12,5 \%$ do total. $\mathrm{O}$ envelhecimento populacional trata-se de uma realidade. As previsões do IBGE apontam, ainda, que de 2000 a 2060 a quantidade de idosos no país aumentará em 417\%(2). Nesse contexto, o aumento da expectativa de vida traz consigo a preocupação com a saúde, pois as doenças ligadas ao processo de envelhecimento podem levar ao aumento dos custos assistenciais de saúde (3).

Segundo Corazza(4), os objetivos de um programa de exercícios para pessoas idosas devem estar diretamente relacionados com benefícios às modificações físicas mais importantes decorrentes do processo de envelhecimento. Sob essa perspectiva, o objetivo principal do treinamento físico deve ser o desenvolvimento e aperfeiçoamento das capacidades físico-motoras (condicionais e coordenativas) do praticante, necessárias para obter bons níveis de rendimento físico(5) para a faixa etária.

Hoje, o esporte na terceira idade é uma realidade, desempenhando um papel importante na prevenção de doenças e na promoção da saúde, contribuindo para um envelhecimento com mais qualidade de vida. 
Os Jogos de Integração do Idoso são jogos coletivos alterados para terceira idade. $\mathrm{O}$ jogo adaptado é o jogo de quadra ou de salão, que teve suas regras normais alteradas, com o intuito de permitir aos atletas condições de participação através da redução da intensidade do jogo. A adaptação do jogo para o idoso permite sua participação, independente de sua aptidão física ou habilidades motoras(6). As regras desses jogos foram aprimoradas por meio da prática e são consenso entre representantes dos estados do Rio Grande do Sul, Santa Catarina e Paraná, formalizadas nas reuniões do fórum da Região Sul(6). Os Jogos de Integração do Idoso apresentam-se como alternativa para a inclusão do idoso na política de esporte e lazer, que segundo a Política Nacional do Idoso(7), é um dos direitos sociais das pessoas com mais de 60 anos. Estes jogos são realizados em muitos municípios do Brasil, normalmente no mês do idoso em outubro, como também, em novembro, para promover a integração.

No Estado do Rio Grande do Sul, entidades governamentais têm oferecido a prática desses jogos à população desde 1998 (6). A prática pelo idoso de jogos adaptados é uma das intervenções importantes no processo do envelhecimento, por suas características lúdicas, socializantes, de variedade de movimentos e exigências posturais e de decisão. Os jogos com bola exigem atenção constante, tanto para acompanhar a trajetória da bola como para adaptar-se rapidamente à jogada, além de memorização de regras(6).

Esta modalidade de atividade física, além de benefícios na capacidade aeróbica, na flexibilidade e na mobilidade oportuniza ao idoso o aprendizado ou a manutenção de habilidades motoras, gerando consequências importantes no comportamento motor, agindo sobre o bem-estar físico, o bem-estar psicológico, a funcionalidade, o social e cognitivo (6).

O objetivo deste estudo foi comparar o nível de aptidão física de idosos participantes de uma equipe esportiva de jogos adaptados para a terceira idade com idosos sedentários.

\section{Métodos}

\section{Desenho de estudo e amostra}

Este estudo caracteriza-se como transversal e analítico para o qual foram convidados para participar os 115 alunos da Universidade Aberta para Terceira Idade (UNATI) do Centro Universitário La Salle (UNILASALLE), em Canoas RS. UNATI é um programa gratuito de extensão comunitária voltado para a terceira idade(8). Os critérios de inclusão foram participar das atividades da UNILASALLE e ter idade igual ou acima de 60 anos. Para ser incluído no grupo Equipe Esportiva (EE) o critério foi praticar os jogos adaptados duas vezes por semana (fisicamente ativos), há pelo menos 6 meses. A amostra do Grupo Sedentário (GS) foi realizada por convite pessoal a participantes das oficinas na UNILASALLE, com mais de 60 anos que estavam presentes nos dias disponíveis para avaliação e que não realizavam nenhuma atividade física habitualmente, nem na UNILASALLE nem em outro local. Os critérios de exclusão foram, para a EE, apresentar baixa assiduidade (menos de dois terços de presença) e para ambos os grupos estar impossibilitado de realizar as avaliações por problemas de saúde nos dias de coleta

\section{Aspectos éticos}

A pesquisa foi aprovada pelo Comitê de Ética em Pesquisa do Centro Universitário UNILASALLE, sob número 5307/2013, seguiu as atualizações da Declaração de Helsinque e o Termo de Consentimento Livre e Esclarecido (TCLE) foi assinado pelos participantes.

\section{Jogos de Integração do Idoso}

Os jogos ministrados aos participantes da UNATI-LASALLE foram o Câmbio Competitivo e o Basquete Relógio com Deslocamento, descritos a seguir.

\section{Câmbio Competitivo}

O Câmbio Competitivo, ou simplesmente Câmbio, trata-se de voleibol adaptado para a terceira idade no qual participam equipes com 12 jogadores, sendo 9 titulares e 3 reservas. $\mathrm{O}$ jogo é realizado em 2 tempos de 15 minutos. Para o desenvolvimento do jogo precisa-se de quadra de voleibol, bola de voleibol e rede em altura de $2,24 \mathrm{~m}$ para equipes femininas e 
$2,43 \mathrm{~m}$ para equipes masculinas. Cada equipe ocupa meia quadra. Os nove jogadores de cada equipe dispostos na meia quadra, de frente para a rede, ocupam os espaços demarcados em posições predeterminadas.

Após o sorteio da posse de bola, o início do jogo é dado pelo jogador da posição 8 (no centro da quadra), quando esse lançar a bola por cima da rede dando a ordem de "Câmbio!".

O saque deverá ser arremessado com a bola presa em uma ou as duas mãos por cima da rede e a recepção através de bola presa, devendo ser executados no máximo 5 passes sendo o $5^{\circ}$ passe para o jogador do centro (posição 8) que ao arremessar a bola para o lado adversário dará a ordem de "Câmbio!", para que ocorra a rotação do time que arremessou a bola.

O rodízio se dá após o jogador passar a bola para a quadra adversária quando todos os jogadores da sua equipe trocam de lugar, no sentido horário em posição numérica crescente. Após o rodízio, sai o jogador da posição 9 e entra o jogador da posição 10 da fileira de reserva para a posição 1 . A reversão da bola acontecerá quando o saque não ultrapassar a rede, ou quando for arremessada para fora da quadra, na situação de saque. Será permitido um pique de bola no chão na recepção do saque.

É executada a pontuação em "Tie Break" somando ao final do tempo o maior número de pontos obtidos. Dá ponto para a equipe adversária quem deixar a bola cair no chão, deixar a bola picar mais de uma vez no chão quando da recepção do saque, não executar corretamente o rodízio, errar o número de passes, errar a posição do arremesso (posição oito), arremessar a bola para a quadra adversária de modo que esta não ultrapasse por sobre a rede, não executar o máximo de 5 passes.

Além dos jogadores são necessários dois técnicos, sendo um para cada time, para auxiliar o desenvolvimento do jogo e quatro facilitadores com as funções de árbitro, mesário e um auxiliar em cada meia quadra com a função de ajudar no rodízio(6).

\section{Basquete Relógio com Deslocamento}

O Basquete Relógio Deslocamento, ou simplesmente Basquete Relógio, como o nome indica, é uma adaptação da modalidade basquetebol. Para sua realização, é necessária uma quadra de basquetebol com 2 tabelas e 2 bolas oficiais, sendo uma cesta e uma bola para cada equipe. Cada equipe é composta de nove jogadores que formarão duas fileiras em frente à cesta, atrás do círculo do garrafão (ou a 5 metros da tabela) defrontando-se a uma distância de 3 metros entre si. Os jogadores da fileira da esquerda, de frente para a tabela, ocuparão as posições de números ímpares, sendo que o primeiro jogador da fila estará ocupando a posição 1 e o último a posição 9 . Os jogadores da fileira da direita, de frente para a tabela, ocuparão as posições de números pares, de 2 a 8 , sendo a posição 2 a do primeiro jogador da fila e a posição de número 8 a do último. $\mathrm{O}$ jogo obedece aos fundamentos do basquete. Estando os jogadores posicionados em fileiras, de frente um para o outro, o jogo inicia com a bola de posse do jogador da posição 9 que a passará para o jogador da posição 8 e assim sucessivamente, sendo a bola passada alternadamente (ou em zig-zag) entre as duas fileiras até chegar ao jogador da posição 1 . O deslocamento tem início com a passagem de bola do jogador da posição 9 para o jogador da posição 8 , que mudará de posição seguindo o movimento da bola. O mesmo acontece com os jogadores de todas as posições, que se deslocarão após passarem a bola. O jogador da posição 1 , ao receber a bola, se deslocará picando a bola em direção à cesta e executará o arremesso. Após o arremesso deve pegar a bola e conduzi-la picando até o final da sua fileira, passando pela esquerda ou por fora das fileiras, até chegar a posição 9 , dando continuidade ao jogo com a passagem da bola para o jogador da posição 8(6).

\section{Senior physical test (SFT) e procedimentos de coleta de dados}

Previamente, foi realizada uma breve apresentação do estudo e respectivos objetivos e descrição dos procedimentos a serem realizados durante a avaliação e, após o aceite em participar da pesquisa foi solicitada a assinatura do TCLE, sendo uma via entregue ao participante.

Para se avaliar a aptidão física dos participantes, utilizou-se um conjunto de testes integrantes do Senior Fitness Test (SFT) elaborada por Rikli e Jones(9). Foram 
selecionadas as seguintes testes : força e resistência de membros inferiores e superiores, capacidade aeróbica, flexibilidade dos membros inferiores e dos membros superiores e velocidade, agilidade e equilíbrio dinâmico.

A força e resistência de membros inferiores (Teste 1), foi avaliada com o teste de sentar e levantar da cadeira (30-Second Chair Stand), pelo número de vezes que o participante levou para levantar e sentar de uma cadeira no período de 30 segundos. A avaliação de força e resistência de membro superior (Teste 2), foi avaliado pelo teste de flexão do braço (Arm Curl), medido pelo número de vezes em que o participante conseguiu flexionar o braço dominante segurando um peso de $2 \mathrm{~kg}$ ajustados dos $(2,27 \mathrm{Kg})$ para mulheres e $4 \mathrm{~kg}$ ajustados dos $(3,63 \mathrm{~kg})$ para homens conforme as referências do protocolo SFT, também em 30 segundos. A avaliação da capacidade aeróbica (Teste 3) foi avaliada pelo teste da marcha estacionária (2-Minute Step Test), em que se conta o número de passos que o participante consegue realizar durante uma marcha estacionária de 2 minutos. $\mathrm{Na}$ sequência, avaliou-se a flexibilidade dos membros inferiores (Teste 4), com o teste de sentar e alcançar ao pé (Chair Sit And Reach); o participante parte de uma posição sentada para frente da cadeira, com a perna estendida e mãos sobrepostas mede-se a distância atingida dos dedos das mãos e pés em cm: (+ou-) entre os dedos estendidos e ponta dos dedos do pé durante 2 segundos. O zero da régua foi direcionado ao pé registrou-se a média entre duas medições. Para se avaliar a flexibilidade dos membros superiores (Teste 5), utilizou-se o teste de alcançar as costas (Back Scratch), foi realizada com o participante em posição ortostática com o braço dominante elevado em flexão e com a palma da mão voltada para o centro das costas e o outro por baixo e atrás das costas durante 2 segundos. Mediu-se a distância em cm (+ou-) entre os dedos médios registrando a média entre as duas medições. Finalmente, para avaliar velocidade, agilidade e equilíbrio dinâmico (Teste 6), utilizou-se o teste de levantar e caminhar (8-Foot Up and $G o$ ), com o participante sentado, solicitou se que o mesmo levantasse, caminhasse $2,44 \mathrm{~m}$ e retornasse a cadeira. O melhor tempo para realizar essa tarefa em duas tentativas foi registrado e usado como avaliação(9).

A EE foi avaliada na sala de ginástica do poliesportivo, e o GS em uma sala de aula na UNILASALLE. A equipe de avaliação que avaliou ambos os grupos EE e GS era formada por um professor de Educação Física e três estagiários acadêmicos em Educação Física, todos foram treinados para padronização das técnicas de mensuração. O protocolo de avaliação para ambos os grupos EE e GS se deu pela montagem de um circuito composto pelos testes ( 1 a 6). Para a realização dos testes foi utilizado um cronômetro, régua de 50 centímetros, cone, cadeira, halteres de $2 \mathrm{~kg}$ para mulheres e $4 \mathrm{~kg}$ para homens. Segundo os critérios de classificação da bateria SFT os grupos foram divididos em faixas de desempenho de aptidão física: normal, acima do normal e abaixo do normal. Conforme a descrição das autoras a atribuição da faixa normal ocorre quando o intervalo de normalidade num respectivo teste for obtido por $50 \%$ dos participantes(10). Aqueles com médias inferiores são considerados abaixo e para os com médias superiores são considerados acima.

Todas as dúvidas foram esclarecidas antes e durante a aplicação das avaliações. Para uma melhor compreensão, cada avaliação foi previamente demonstrada e uma experiência de familiarização foi executada por parte de cada participante antes do momento de avaliação.

\section{Análise estatística}

Realizou-se análise descritiva das variáveis antropométricas da altura e peso e analítica quanto à aptidão física composta de força e resistência de membros inferiores, força e resistência de membro superior, capacidade aeróbica, flexibilidade de membros inferiores, flexibilidade de membros superiores, agilidade, entre os grupos EE e GS. O percentual para faixa de normalidade foi calculado para os seis, conforme prescrito na metodologia do $\operatorname{SFT}(9,10)$. As médias dos desempenhos nos testes foram comparadas entre os dois grupos e testadas pelo $t$ de Student não pareado e teste de Bartlett quanto à normalidade na distribuição dos dados. A distribuição percentual dos homens e mulheres participantes nos grupos foi testada pelo teste 
exato de Fischer, visto que o número de homens esperado em cada grupo foi menor que cinco. O nível de confiança adotado foi de $95 \%$ em todas as análises. Utilizou-se o programa estatístico Epi Info $^{\mathrm{TM}} 7$ para realizar as análises.

\section{Resultados}

Depois de aplicados os critérios de exclusão, participaram do estudo 53 pessoas. A Tabela 1 mostra a distribuição dos participantes do estudo segundo sexo nos grupos EE e GS.

Tabela 1 - Distribuição segundo sexo nos grupos Equipe Esportiva (EE) e grupo sedentário (GS) dos idosos da UNATI UNILASALLE $(\mathrm{N}=53)$

\begin{tabular}{lccccc}
\hline Sexo & \multicolumn{2}{c}{ EE } & \multicolumn{2}{c}{ GS } & \\
& $\mathrm{n}$ & $\%$ & $\mathrm{n}$ & $\%$ & $P$ \\
\hline \hline Masculino & 5 & $(18 \%)$ & 2 & $(8 \%)$ & 0,426 \\
Feminino & 23 & $(82 \%)$ & 23 & $(92 \%)$ & \\
Total & 28 & $(53 \%)$ & 25 & $(47 \%)$ & \\
\hline
\end{tabular}

$\boldsymbol{P}$ : p-valor resultado do teste de Fischer.

Conforme observa-se na Tabela 2, não houve diferença estatística entre os dois grupos quanto à idade, estatura e peso.

O desempenho nos testes de aptidão física foi melhor na EE comparando-se com o GS, sendo que houve diferença significativa em flexibilidade de membros superiores (alcance das costas e flexão dos braços), agilidade e equilíbrio dinâmico (levantar e caminhar), capacidade aeróbica (marcha estacionária) e flexibilidade dos membros inferiores, principalmente à esquerda.

As Figuras 1 e 2 apresentam os resultados dos testes segundo a classificação preconizada pelo $\operatorname{SFT}(9,10)$ : "Normal", "Acima do Normal" e "Abaixo do Normal".

Os resultados demonstram que ao somar a faixa normal com a faixa acima do normal, observam-se níveis ainda mais elevados de desempenho físico na EE em comparação com o GS: Em relação à força e resistência de membros inferiores observou-se que $89,3 \% \mathrm{em}$ EE estavam nas faixas normal e acima do normal e $72 \%$ em GS nessas mesmas faixas. Em força e resistência de membro superior, $67,8 \%$ em EE e $64 \%$ em GS. Quanto à capacidade aeróbica, nessas faixas, estavam $78,5 \%$ em EE $24 \%$ em GS, destaca-se que $76 \%$ foram classificados em GS como abaixo do normal. A diferença estatisticamente significativa entre os desempenhos de EE e GS nos Testes 1,2 e 3, mostrou-se somente na marcha estacionária (Teste 3$)(\mathrm{p}<0,001)$.

Na Figura 2, observa-se diferenças entre EE e GS nos testes 4,5 e 6 . O percentual de desempenho dentro da faixa considerada normal e acima do normal foi maior para a EE. Ao somar a faixa normal com a faixa acima do normal obtemos resultados ainda maiores para EE. Nos testes 4 e 5 que avaliam a flexibilidade a EE mostra medidas em sentar e alcançar até os pés esquerdo e direito entre $85,7 \%$ e $89,3 \%$ respectivamente, enquanto que o GS teve $68 \%$ e $64 \%$ respectivamente. No teste 6 de levantar e caminhar, na EE somando-se a faixa normal e acima do normal resultou em: $100 \%$ dos participantes e o GS: $56 \%$. Houve diferença estatisticamente significativa nos três testes: flexibilidade de membro inferior esquerdo (Teste 4) $(\mathrm{p}=0,012)$, nos membros superiores (Teste 5) $(\mathrm{E} \mathrm{p}=0,0029)$ e $(\mathrm{D} p=0,0081)$ e na velocidade, agilidade/equilíbrio dinâmico (teste 6) $(\mathrm{p}=0,042)$.

\section{Discussão}

O presente trabalho buscou comparar o nível de aptidão física entre participantes do programa de atividades físicas para a terceira idade (Jogos de Integração para Idosos) e idosos sedentários. Em todas as avaliações físicas, o desempenho normal e acima do normal foi mais elevado nos idosos fisicamente ativos em comparação aos idosos sedentários. Estudo prévio, em idosos fisicamente ativos, praticantes de hidroginástica apresentaram resultados semelhantes aos da EE, ainda assim com menores percentuais, exceto no teste flexão do braço em que obtiveram percentuais superiores $(77,78 \%)(11)$. Destacam-se as evidências significativas obtidas pela marcha estacionária em que $78,5 \%$ dos participantes EE mostraram melhor capacidade aeróbica que os GS ao completarem uma média de 95 passos em comparação a média de 60 passos do GS, respectivamente. Estes achados estão alinhados com a literatura quanto aos benefícios da prática de atividade física para a melhoria do condicionamento cardiorrespiratório(6). Segundo Jones e Rikli(10), valores inferiores a 65 passos são 
Tabela 2 - Distribuição segundo sexo nos grupos Equipe Esportiva (EE) e grupo sedentário (GS) dos idosos da UNATI UNILASALLE $(\mathrm{N}=53)$

\begin{tabular}{|c|c|c|c|}
\hline Característica / Avaliação & $\begin{array}{c}\text { EE } \\
\text { Média } \pm \mathbf{D P}\end{array}$ & $\begin{array}{c}\text { GS } \\
\text { Média } \pm \text { DP }\end{array}$ & $P * *$ \\
\hline Idade (anos) & $266,5 \pm 4,51$ & $688,4 \pm 5,33$ & 0,1568 \\
\hline Peso (Kg) & $68,7 \pm 14,41$ & $71,2 \pm 13,62$ & 0,5301 \\
\hline Altura (m) & $1,58 \pm 0,07$ & $1,55 \pm 0,08$ & 0,2249 \\
\hline \multicolumn{4}{|l|}{ Força e Resistência MI (Teste 1) } \\
\hline Levantar da Cadeira (rep) & $13,42 \pm 2,28$ & $12,44 \pm 3,30$ & 0,2070 \\
\hline $\begin{array}{c}\text { (Teste 2) Força e Resistência MS } \\
\text { Flexão do Braço Dominante }\end{array}$ & $19,28 \pm 4,36$ & $18,36 \pm 5,43$ & 0,2050 \\
\hline $\begin{array}{c}\text { (Teste 3) Capacidade aeróbica } \\
\text { Marcha Estacionária (rep) }\end{array}$ & $95,10 \pm 26,62$ & $60,80 \pm 21,38$ & $<, 0001$ \\
\hline $\begin{array}{l}\text { (Teste 4) Flexibilidade MI } \\
\quad \text { Sentar e Alcançar os Pés E (cm) }\end{array}$ & $3,42 \pm 7,76$ & $-2,80 \pm 9,62$ & 0,0120 \\
\hline Sentar e Alcançar os Pés D $(\mathrm{cm})$ & $1,78 \pm 7,74$ & $-2,80 \pm 9,97$ & 0,0659 \\
\hline $\begin{array}{l}\text { (Teste 5) Flexibilidade } \mathbf{M S} \\
\text { Alcançar as Costas E (cm) }\end{array}$ & $-6,92 \pm 10,42$ & $-16,84 \pm 12,63$ & $\mathbf{0 , 0 0 2 9}$ \\
\hline Alcançar as Costas D $(\mathrm{cm})$ & $-3,89 \pm 10,92$ & $-13,16 \pm 13,52$ & 0,0081 \\
\hline
\end{tabular}

(Teste 6) Velocidade, agilidade e equilíbrio dinâmico

Levantar e Caminhar (seg)

$0,05 \pm 0,00 \quad 0,13 \pm 0,21$

0,0428

Descrição das características físicas antropométricas e resultados dos testes físicos da bateria Senior Fitness Test da Equipe Esportiva (EE) e do Grupo Sedentário (GS). Força e Resistência Membros Inferiores (MI), Força e Resistência Membros Superiores (MS), Flexibilidade Membros Inferiores (MI), Flexibilidade Membros Superiores (MS), rep. = repetições, seg. = segundos, DP: desvio padrão, D: direito(a), E=esquerdo(a), P: p-valor resultados do teste $t$ de Student.

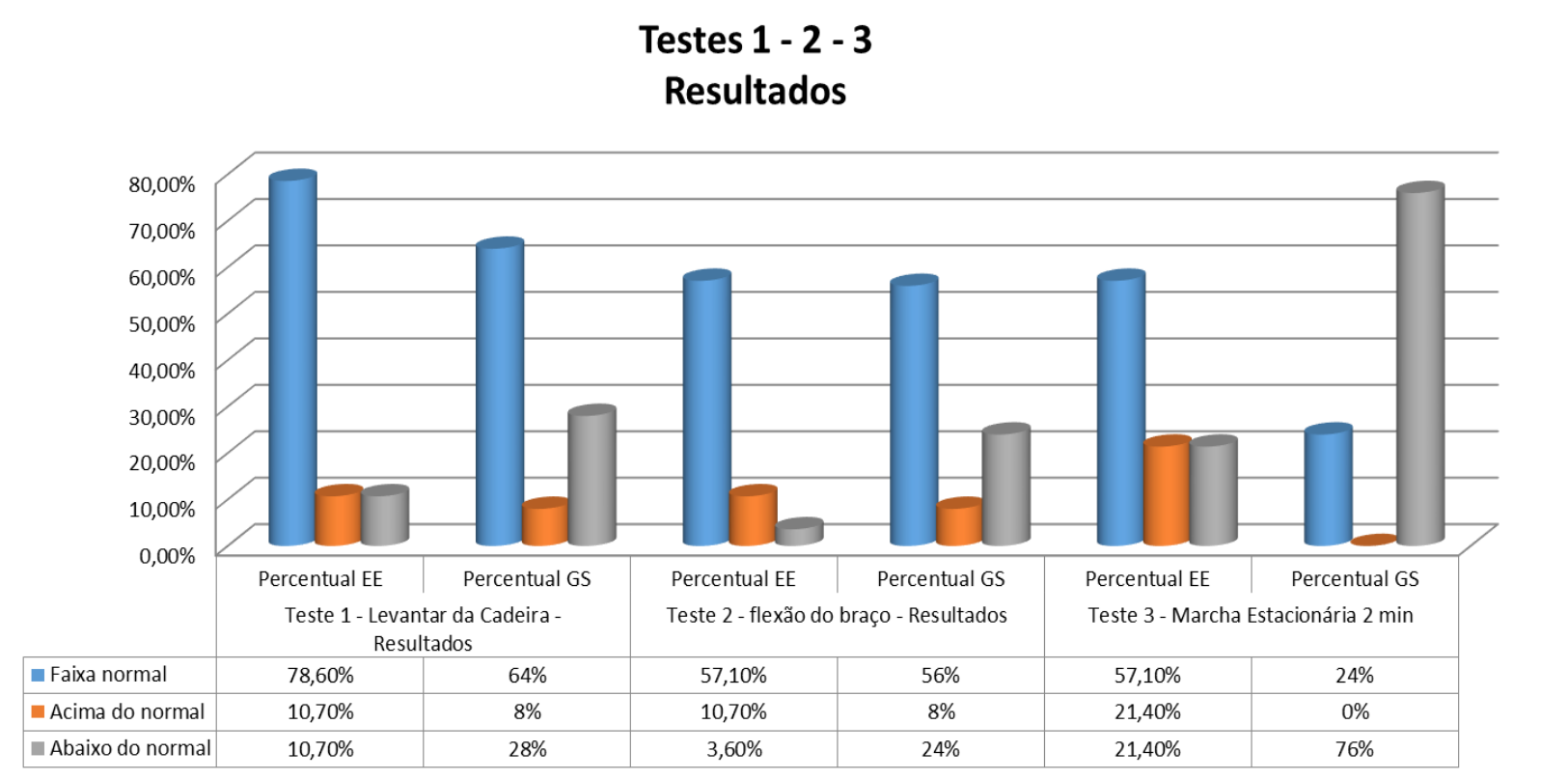

Figura 1 - Teste 1 levantar e sentar da cadeira (força e resistência de membros inferiores), Teste 2 flexão do braço (força e resistência de membro superior) e Teste 3 marcha estacionária (capacidade aeróbica). Percentual da classificação para normalidade da capacidade funcional. Percentagem da Equipe Esportiva (EE), Grupo Sedentário (GS) e faixa de normalidade igual a 50\%. 




Figura 2 - Teste 4 - Sentar e alcançar o pé (flexibilidade de membros inferiores), Teste 5 - Alcançar as costas (flexibilidade de membros superiores) e Teste 6 - Levantar e caminhar (velocidade, agilidade e equilíbrio dinâmico). Percentual da classificação para normalidade da capacidade funcional. Percentagem da equipe esportiva (EE), grupo sedentário (GS) e faixa de normalidade $50 \%$.

representativos de entrada em zona de risco para esta condição funcional em pessoas idosas. O mesmo se aplica para a velocidade, agilidade e equilíbrio dinâmico em que a diferença significativa para a EE mostrou que $100 \%$ dos idosos ativos tem este nível de desempenho melhor que o GS. A EE ao completar em média 0,05 seg não se encontra em zona de risco para esta capacidade funcional contrariando os $0,13 \mathrm{seg}$ do GS que não deveria ultrapassar $0,9 \mathrm{seg}(10)$.

Rikli e Jones(9) apontam que, em aptidão física, a manutenção da força muscular deve ser a prioridade com idosos, pois, o declínio da força muscular pode acompanhar a sarcopenia após os 50 anos. Essa perda ocorre entre 15 a $20 \%$ por década(12,13). A diminuição da função muscular, associada ao avançar da idade, pode produzir efeitos negativos na capacidade de desempenho de atividades cotidianas, como subir degraus, caminhar, levantar da cadeira entre outras(13-15). Preservar a força também é importante para a redução dos riscos de quedas e suas lesões relacionadas $(16,17)$. No contexto de manutenção da força muscular, essencial durante o processo de envelhecimento, a mensuração deste componente da aptidão física assume um papel importante na elaboração de programas de atividade física para idosos. Os idosos praticantes de jogos adaptados da equipe esportiva mostraram melhor desempenho nos testes que avaliaram a aptidão física dos membros inferiores em comparação com os idosos sedentários da UNATI. A força e resistência ainda que não tenha tido uma diferença significativa foi mais expressiva na equipe esportiva em que $89,3 \%$ tiveram melhor aptidão dos membros inferiores que $72 \%$ no grupo sedentário. Além disso o grupo sedentário mostrou mais que o dobro de idosos abaixo da normalidade $28 \%$ resultado que expressa declínio no padrão de força. Medidas abaixo da normalidade estão associadas a maiores riscos para a saúde(9).

Quanto à força muscular de membros superiores, a maioria dos participantes do estudo apresentaram normal ou acima do normal $(67,8 \%$ EE e $64,0 \%$ GS) (Figura 1) resultados semelhantes ao do estudo de Elias et al. (11), no qual $77,78 \%$ dos participantes apresentaram na faixa normal. A pequena diferença no desempenho da EE e do GS foi estatisticamente significativa que pode ser atribuída à consistência e especificidade do conteúdo do treinamento esportivo, que não contemplava tarefas dos indivíduos que não praticavam atividade física com regularidade.

Quanto à capacidade aeróbica, importante capacidade para que as pessoas consigam 
realizar as tarefas do dia a dia como: caminhar, fazer compras, participar de atividades esportivas ou recreativas, houve diferença significativa (Figura 1 ) entre a EE $(78,5 \%)$ a o GS (24,0\%). Se comparados com o estudo de Elias et al.(11) que encontraram 55,56\% entre os praticantes de hidroginástica, identifica-se uma correspondência bastante mais elevada de percentual com a equipe esportiva pelos jogos adaptados. Nesse contexto, a literatura mostra que o aumento do nível de atividade física pode levar a melhoras substanciais da capacidade aeróbica de idosos(13).

Quanto à flexibilidade, foi possível observar diferenças significativas tanto nos membros superiores como nos membros inferiores a favor da equipe esportiva. Estas diferenças resultam dos exercícios específicos realizados na prática das modalidades Câmbio e Basquete Relógio, que estimulam maior mobilidade e amplitude articular do ombro e flexibilidade da passada. A flexibilidade é um componente neuromotor indispensável nos programas de exercício para pessoas idosas(13).

Agilidade combinada (velocidade e coordenação) e equilíbrio dinâmico (manutenção da estabilidade postural durante o movimento) são aspectos importantes para várias tarefas comuns de mobilidade que exigem manobras rápidas, como subir e descer de ônibus de maneira satisfatória, desviar de um carro ou de outro objeto em movimento, levantar-se a tempo para atender ao telefone, ir ao banheiro ou fazer algo na cozinha. Além disso, estas qualidades físicas são necessárias para a participação segura em muitos esportes e jogos recreativos. Embora alguns possam argumentar que agilidade/equilíbrio dinâmico representem dois componentes diferentes da aptidão física e que devem ser avaliados separadamente, para Rikli e Jones(9) essas valências são tratadas como medida composta, uma vez que devem trabalhar em conjunto para desempenho satisfatório de muitas atividades diárias, como as mencionadas anteriormente.

No teste levantar e caminhar, contrariamente ao grupo sedentário todos os participantes da equipe esportiva apresentaram-se na faixa normal e acima do normal. O resultado pode ser explicado devido às características dos jogos adaptados: Basquete Relógio e Câmbio). Possivelmente as atividades desenvolvidas com a equipe proporcionam estímulos músculos esqueléticos e proprioceptivos, que juntos contribuem para um melhor controle postural, podendo desenvolver força e especialmente agilidade, evidenciado nos resultados dos testes de aptidão física.

Os resultados obtidos no presente estudo permitiram conhecer o nível de aptidão física dos alunos participantes de uma equipe esportiva de jogos adaptados para a terceira idade. Estas evidências indicam que idosos de tais níveis funcionais podem conservar reservas funcionais fisiológicas e manter um bom nível de capacidade física motora $\mathrm{e}$ funcional, independente da idade cronológica.

\section{Pontos fortes e limitações do estudo}

Dentre os pontos fortes do estudo, destacamse três. O primeiro refere-se ao tempo de prática. O tempo de participação em um programa e os resultados obtidos reforça a ideia de que a prática sistemática de exercício físico na terceira idade, ao longo do tempo, promove o desempenho das tarefas associadas às valências físicas.

$\mathrm{O}$ segundo refere-se à especificidade do treino, o efeito do treinamento se mantém no idoso e as capacidades avaliadas são essenciais no desempenho dos jogos, nas atividades da vida diária, na prevenção de acidentes como as quedas por exemplo.

O terceiro está relacionado ao processo de declínio das capacidades no envelhecimento. A participação em um programa de exercícios físicos não só melhora as capacidades físicas, mas também pode prevenir as perdas físicas do processo natural do envelhecimento.

Sob essa perspectiva, um programa que atenda o quadro completo do envelhecimento tem como característica procurar intervir sobre a aptidão física funcional, função cognitiva e sócio afetiva das pessoas idosas. Nesse sentido, os programas com jogos adaptados, como é o caso do Câmbio e do Basquete Relógio, incluem-se no paradigma "dual task" (dupla tarefa) em que há um envolvimento físico e mental estimulante que desafia em simultâneo as capacidades físicas (condicionais e coordenativas) junto com habilidades mentais que neles se destacam: a atenção, velocidade de reação, memória e tomada de decisão (funções executivas), 
indispensáveis na prática de jogos coletivos. Assim sendo, a ausência do controle de variáveis cognitivas neste estudo aparece como uma limitação que merece ser discutida. Os jogos em equipe que buscam um mesmo objetivo levam a ativar o uso de diversas habilidades cognitivas, destacam-se a atenção visuo-espacial, seletiva, dividida, a velocidade comportamental (velocidade de processamento informacional), e um maior empenho da flexibilidade mental como, organização, execução, capacidade decisional (funções executivas). No entanto, ainda são poucos os estudos realizados utilizando os jogos coletivos, que exploram este campo integrado de análises. Sobre os efeitos positivos de modalidades esportivas na cognição de pessoas idosas, Spirduso demonstrou associação positiva entre prática esportiva (tênis e handebol) e velocidade de processamento informacional(18). Recentemente um outro estudo mostrou a contribuição dos efeitos de jogos tradicionais e cognitivos na saúde mental de idosos (19). Esta combinação de capacidades é essencial nas atividades físicas e mentais necessárias as exigências motoras e cognitivas do dia-a-dia. Numa perspectiva preventiva e de promoção da independência, autonomia, segurança e saúde, vários autores sugerem os procedimentos de dupla-tarefa em programas de atividade física visando a prevenção de quedas(20-25).

\section{Conclusão}

Este estudo buscou estimar os benefícios da prática de atividade para a aptidão física em idosos da UNATI UNILASALLE, que participaram de um programa de jogos adaptados. Os resultados mostraram que aqueles que apresentaram frequência de pelo menos duas vezes por semana, mostraram melhores níveis de aptidão física que os idosos sedentários. Estes achados indicam que o programa de atividades físicas para idosos tem contribuído para melhorar a aptidão física dos participantes regulares.

Recomenda-se que sejam identificadas as necessidades individuais ou de um grupo, para que cada componente da aptidão física seja acompanhado e planejado em jogos adaptados, atividades recreativas, atividades da vida diária entre outras para que todas as capacidades sejam desenvolvidas.

Sugere-se explorar habilidades cognitivas em estudos futuros. Adicionalmente, mais informações sobre os benefícios deste tipo de programa podem ser exploradas nas dimensões sócio emocionais, relacionais e autoestima.

\section{Agradecimentos}

Os autores agradecem à coordenadora da UNATI La Salle, Professora MS Juliana Ludwig Justo e aos alunos da UNATI.

\section{Declaração de conflito de interesses}

Não há nenhum conflito de interesses em relação ao presente estudo.

\section{Declaração de financiamento}

Não houve financiamento para o presente estudo.

\section{Referências}

1. Brasil, Lei 10741/03 Estatuto do Idoso. [Internet]. Brasil 2016. [Acesso em 28 Oct. 2016] http://www.presrepublica.jusbrasil.com.br/ legislacao/98301/estatuto-do-idoso-lei10741-03

2. Instituto Brasileiro de Geografia e Estatística. Projeção de População, revisão 2013. [Internet]. Brasil 2016. [Acesso em 2 Aug. 2017] http://www.ibge.gov.br

3. Papalia DE, Olds SW, Feldman RD. Desenvolvimento humano. $10^{\mathrm{a}}$ ed. Porto Alegre: AMGH, 2009.

4. Corazza MA. Terceira idade e atividade física $2^{\mathrm{a}}$ ed. Local: Phorte Editora Ltda., 2006.

5. Barbanti VJ. Teoria e prática do treinamento esportivo $2^{\text {a }}$ ed. São Paulo: Editora Edgard Blücher Ltda; 1997.

6. SEL: Secretaria Estadual do Esporte e do Lazer do Rio Grande do Sul. Regulamento oficial do XV Jogos de Integração do Idoso do RS, ano 2013. [Internet]. Brasil 2016. [Acesso em 28 Oct. 2016] http://www.sel.rs.gov.br/upload/13778683 27_Regulamento\%20oficial\%20\%20XV\% $20 \mathrm{~J}$ ogos\%20de $\% 20$ Integracao $\% 20 \mathrm{do} \% 20 \mathrm{I}$ 
doso $\% 20 \mathrm{do} \% 20 \mathrm{RS} \% 202013 \% 2030-08$ 2013.

7. Brasil, Lei 8842/94. Política nacional do idoso. [Internet]. Brasil 2016. [Acesso em 280ct.2016]

http://www.planalto.gov.br/ccivil_03/Leis/ L8842.htm

8. UNILASALLE. UNATI. [Internet]. Brasil 2016. [Acesso em 28 Oct. 2016] http://www.unilasalle.edu.br/canoas/unati/

9. Rikli R, Jones J. Development and validation of a functional fitness test for community-residing older adults. Journal of Aging and Physical Activity. 1999;7:129161. doi.org/10.1123/japa.7.2.129

10. Jones CJ, Rikli RE. Measurin functional. Journal on Active Aging. 2002; 25-30.

11. Elias RMG, Gonçalves ECA, Moreira ACF, Formaggio C, Fernandes CAM. Aptidão Física funcional de idosos praticantes de hidroginástica. Revista Brasileira de Geriatria e Gerontologia. [Online] 2012;15 (1): 79-86

12. Ribeiro LHM, Neri AL. Exercícios físicos, força muscular e atividades de vida diária em mulheres idosas. Revista Ciência e Saúde Coletiva. 2012; 17 (8): 2169-2180. doi.org/10.1590/S1413-

81232012000800027

13. Garber CE, Blissmer B, Deschenes MR, Franklin BA, Lamonte MJ, Lee I-M, et al. American College of Sports Medicine position stand. Quantity and quality of exercise for developing and maintaining cardiorespiratory, musculoskeletal, and neuromotor fitness in apparently healthy adults: guidance for prescribing exercise. Medicine and Science in Sports and Exercise. [Online] 2011;43(7): 1334-1359. Doi.org/10.1249/MSS.0b013e318213fefb

14. Kaneko M, Morimoto Y, Kimura M, Fuchimoto K, Fuchimoto T. A kinematic analysis of walking and physical fitness testing in elderly women. Canadian Journal of Sports Sciences. [Online] 1991;16: 223228.
15. Millington PJ, Myklebust BM, Shambes GM. Biomechanical analysis of the sit-tostand motion in elderly persons. Archives of Physical Medicine and Rehabilitation. 1992;73:609-617.

16. Lord SR, Ward JA, Williams P, Strudwick $M$. The effects of a 12-month exercise trial on balance, strength, and falls in older women: a randomized controlled trial. Journal of the American Geriatrics Society. [Online] 1995;43:1198-1206.

17. Streit IA, Mazo GZ, Virtuoso JF, Menezes EC, Gonçalves E. Aptidão física e ocorrência de quedas em idosos praticantes de exercícios físicos. Revista Brasileira de Atividade Física \& Saúde. 2011; 16 (4): 346-352.

doi.org/10.12820/rbafs.v.16n4p346-352

18. Spirduso WW. Reaction and movement time as a function of age and physical activity level. Journal of Gerontology. [Online] 1975;30(4), 435-340.

19. Pinheiro S. O impacto de um programa de jogos tradicionais e de um programa de jogos cognitivos nas funções cognitivas, na qualidade de vida e nas relações sociais de idosos. Repositório UTAD. Dissertação de Mestrado.URI:

http://hdl.handle.net/10348/7489. 2017.

20. Pellecchia GL. Dual-task training reduces impact of cognitive task on postural sway. Journal of Motor Behaviour. [Online] 2005;37(3), 239-246. Available from: doi.org/10.3200/JMBR.37.3.239-246

21. Robertson MC, Gillespie LD. Fall prevention in community-dwelling older adults. JAMA. [Online] 2013;309(13):14061407. doi:10.1001/jama.2013.3130.

22. Soares GS, Peyré LA. Parkinson's disease and physical exercise: a literature review. Ciência em Movimento. 2010;24:69-83.

23. Vaillant J, Vuillerme $\mathrm{N}$, Martigné $\mathrm{P}$, Caillat-Miousse JL, Parisot J, Nougier V, Juvin R. Balance, aging, and osteoporosis: effects of cognitive exercices combined with physiotherapy. Joint Bone Spine. 2006;73(4):414-418. doi.org/10.1016/j.jbspin.2005.07.003 
24. Silsupadol P, Shumway-Cook A, Lugade V, van Donkelaar P, Chou LS, Mayr U, et al. Effects of single-task versus dual-task training on balance performance in doubleblin, randomized controlled trial. Archives of Physical Medicine and Rehabilitation. 2009;90(3):381-387.

doi.org/10.1016/j.apmr.2008.09.559.

25. Pesce C, Cereatti L, Casella R, Baldari C, Capranica L. Preservation of visual attention in older expert orienteers at rest and under physical effort. Journal of Sport and Exercise Psychology. 2007;29:78- 99. 